\title{
Geschlechterunterschiede in den Arbeitswerten: eine Analyse für die alten Bundesländer 1980-2000
}

\author{
Matthias Pollmann-Schult
}

Angenommen: 21. Januar 2009 / Online veröffentlicht: 13. März 2009

(C) Institut für Arbeitsmarkt- und Berufsforschung 2009

Zusammenfassung In dem Beitrag wird die Entwicklung der Arbeitswerte von Frauen und Männern zwischen 1980 und 2000 anhand der Daten des ALLBUS untersucht. Es wird damit der Frage nachgegangen, inwiefern geschlechtsspezifischen Arbeitswerten eine eigenständige Bedeutung für die Genese von beruflicher Segregation zukommt. Die empirischen Analysen zeigen, dass Männer mehr Wert auf extrinsische Arbeitsaspekte legen, während Frauen altruistische Aspekte höher schätzen. Allerdings sind diese Unterschiede in den Arbeitswerten eher schwach ausgeprägt und haben sich über die Zeit hinweg stark verringert. Während zu Beginn der 1980er-Jahre noch signifikante Unterschiede bestehen, indem Männer mehr Wert auf gute Verdienstmöglichkeiten sowie hohe Aufstiegschancen legen und Frauen die Ausübung einer Tätigkeit mit helfendem Charakter höher schätzen, sind markante Geschlechterunterschiede im Jahr 2000 nur hinsichtlich der altruistischen Arbeitswerte zu beobachten. Die insgesamt eher geringen Geschlechterunterschiede deuten darauf hin, dass angebotsseitige Erklärungsansätze von beruflicher Segregation - dazu gehören neoklassische Ansätze und Sozialisationstheorien - keinen gewichtigen Beitrag zur Erklärung der Arbeitsmarktsegregation leisten können.

M. Pollmann-Schult $(\varangle)$

Universität Bielefeld, Fakultät für Soziologie,

Postfach 100131, 33501 Bielefeld, Deutschland

E-Mail: matthias.pollmann-schult@uni-bielefeld.de

Tel.: +49-521-1064610
Gender differences in job values:

an analysis for western Germany 1980-2000

Abstract This research examines changes in job values of women and men from 1980 to 2000 using data from the ALLBUS. Here we address the question of whether gender differences in job values are of particular importance to explain sex segregation in the labour market. Our results suggest that men tend to place a higher value on extrinsic rewards and women attach greater importance to altruistic rewards than men do. However, these gender differences are limited and have narrowed in recent years. In the early $1980 \mathrm{~s}$ men attached more importance to income and chances of promotion than women did and women indicated a stronger preference to having opportunities to help others, whereas in 2000, we observe gender differences only with regard to altruistic job values. The relatively small gender differences in job values suggest that supply-side explanations for sex segregation - derived from neoclassical and socialisation theory - do not significantly contribute to explain sex segregation in the labour market.

\section{Einleitung}

Die Erwerbschancen von Frauen haben sich in den letzten Jahrzehnten stark verbessert, allerdings erzielen weibliche Erwerbstätige auch heute noch ein deutlich niedrigeres Einkommen als männliche. Dieser Lohnabstand zwischen den Geschlechtern resultiert nicht zuletzt aus der geschlechtsspezifischen Segregation des Arbeitsmarktes, durch die Frauen oftmals in Berufsfeldern mit vergleichsweise niedriger Entlohnung und geringen Aufstiegschancen beschäftigt sind (siehe Achatz et al. 2005; Brückner 2004; Hinz u. Gartner 2005; Liebeskind 2004; Solga u. Konietzka 
2000; Trappe 2006). Während eine Vielzahl von Studien die Folgen beruflicher Segregation untersucht, kommt deren ursächlichen Faktoren nur wenig Aufmerksamkeit zu. Mögliche Ursachen von geschlechtsspezifischer Segregation und den damit einhergehenden Lohnunterschieden zwischen Männern und Frauen finden sich sowohl auf der Nachfrage- als auch Angebotsseite des Arbeitsmarktes (England 2005; Reskin 1993; Reskin u. Bielby 2005). Auf der Nachfrageseite kann berufliche Segregation aus diskriminierenden Einstellungs- und Allokationsentscheidungen der Arbeitgeber resultieren, die dazu führen, dass Frauen auf schlechter bezahlte und statusniedrigere Berufspositionen als gleich qualifizierte Männer platziert werden (siehe Allmendinger u. Hinz 2007). Angebotsseitige Erklärungsansätze - hierzu zählen neoklassische Ansätze und Sozialisationstheorien - betrachten dagegen berufliche Segregation als Ausdruck unterschiedlicher Arbeitswerte und -präferenzen. Während der neoklassische Ansatz geschlechtsspezifische Segregation darauf zurückführt, dass Frauen in einer rationalen Entscheidungssituation geringer entlohnte, aber dafür familienkompatible Berufe wählen, sieht der Sozialisationsansatz die primäre Ursache von Arbeitsmarktsegregation in der geschlechtsspezifischen Vermittlung von Wertorientierungen bei Jungen und Mädchen. Die Frage nach möglichen Geschlechterunterschieden in den Arbeitswerten ist somit zentral für die Analyse der Ursachen von beruflicher Segregation. Insofern gravierende Unterschiede zwischen Männern und Frauen existieren, wären ihre ungünstigere Einkommenssituation und Berufsstellung nicht allein auf diskriminierende Einstellungs- und Beförderungspraktiken der Arbeitgeber zurückzuführen, sondern müssten zumindest partiell als Konsequenz des eigenen Berufswahlverhaltens begriffen werden (Padavic $u$. Reskin 2002).

Obwohl in beiden angebotsseitigen Erklärungsansätzen der Annahme geschlechtsspezifischer Arbeitswerte eine zentrale Rolle zukommt, wurde sie - zumindest für Deutschland - bislang kaum einer empirischen Prüfung unterzogen. Die Ergebnisse empirischer Studien für andere Länder - insbesondere die USA - zeichnen ein eher uneinheitliches Bild. Zwar finden sich Belege dafür, dass Frauen und Männer unterschiedliche Werteeinstellungen aufweisen und Männer stärker zu materiellen Werten neigen als Frauen (siehe etwa Beutel u. Marini 1995), jedoch zeigt sich keine klare empirische Evidenz für Geschlechterdifferenzen hinsichtlich der Arbeitswerte (siehe Halaby 2003; Marini et al. 1996; Rowe u. Snizek 1995; Tolbert u. Moen 1998). ${ }^{1}$

\footnotetext{
${ }^{1} \mathrm{Da}$ im internationalen Vergleich einige Differenzen bezüglich der Geschlechterunterschiede in den Arbeitswerten bestehen und Deutschland diesbezüglich erheblich von anderen europäischen Ländern abweicht (De Vaus u. McAllister 1991), sind die Ergebnisse für die USA nicht problemlos auf Deutschland übertragbar.
}

Für Deutschland existiert meines Wissens bislang keine Studie, die sich explizit mit Geschlechterunterschieden in den Arbeitswerten befasst. In ihrer Analyse der Determinanten von Arbeitswerten konstatieren Braun u. Borg (2004) allerdings bei Männern eine stärkere extrinsische Orientierung als bei Frauen, wogegen die international vergleichende Studie von De Vaus u. McAllister (1991) für Deutschland keine signifikanten Geschlechterunterschiede feststellen. $^{2}$

Die überwiegende Mehrheit der publizierten Studien zu Geschlechterdifferenzen in den Arbeitswerten betrachtet die einzelnen Arbeitsaspekte separat voneinander und untersucht, inwiefern Frauen und Männer den jeweiligen Jobcharakteristika eine ähnliche oder signifikant unterschiedliche Bedeutung beimessen. Hier liegt implizit die Annahme zugrunde, dass die Wichtigkeit der einzelnen Jobeigenschaften handlungsweisend ist, also beispielsweise eine höhere Wertschätzung des Einkommens auf Seiten der Männer sich in einer entsprechenden Berufswahl niederschlägt und dadurch geschlechtsspezifische Einkommensabstände generiert. In der Werteforschung herrscht jedoch Uneinigkeit darüber, ob Handlungsentscheidungen durch solche Wertorientierungen oder aber vielmehr durch das Verhältnis konkurrierender Werte zueinander geprägt werden. Im letzteren Fall würde das Berufswahl- und Arbeitsmarktverhalten auf der Rangordnung der Arbeitsaspekte beruhen. Obwohl sogenannte Rangordnungsverfahren in der Werteforschung eine prominente Rolle einnehmen, wurde dieses Verfahren in der Analyse von Arbeitswerten bislang kaum angewandt (eine Ausnahme stellt Halaby 2003 dar). Um ein möglichst umfassendes Bild hinsichtlich der Geschlechterunterschiede in den Arbeitswerten zu gewinnen, werden in dieser Studie sowohl Rating-Angaben als auch Ranking-Angaben zur Analyse der Arbeitswerte genutzt.

\section{Ursachen geschlechtsspezifischer Arbeitswerte}

Unter Arbeitswerten werden Vorstellungen über die Erwünschtheit unterschiedlicher Arbeitsaspekte verstanden. Diese Arbeitsaspekte beziehen sich auf Arbeitsgratifikationen wie etwa das Erwerbseinkommen oder das Berufsprestige einer Tätigkeit. Zumeist wird zwischen intrinsischen und extrinsischen Gratifikationen differenziert. Extrinsische Gratifikationen stellen Mittel zur Erreichung arbeitsfremder Ziele dar; hierzu zählen neben Einkommen und Berufsprestige ebenfalls die Aufstiegschancen, Arbeitsplatzsicherheit, Arbeitszeitregelung sowie die Arbeitsbelastung. Intrinsische Gratifikationen beziehen sich

\footnotetext{
${ }^{2}$ Darüber hinaus analysieren unter anderem Habich (1986), Borg et al. (1993) sowie Adler u. Brayfield (1997) Arbeitswerte in Deutschland, jedoch werden Geschlechterunterschiede in diesen Studien nicht untersucht.
} 
auf Aspekte, die aus der Arbeit selbst herrühren. Dazu gehören das Interesse an der Arbeit an sich, die Möglichkeit, eigene Fähigkeiten und Kompetenzen adäquat anzuwenden, sowie selbstbestimmtes Arbeiten. Den intrinsischen Gratifikationen werden ferner sowohl altruistische Gratifikationen - wie etwa die Möglichkeit, anderen Personen zu helfen oder eine für die Gesellschaft sinnvolle Tätigkeit auszuüben - als auch soziale Gratifikationen - etwa die Möglichkeit, mit anderen Menschen zu arbeitenzugerechnet.

Angebotsseitige Erklärungsansätze begründen berufliche Segregation mit Unterschieden im Berufswahlverhalten (einen umfassenden Theorieüberblick bietet Achatz 2005). Gemäß dieser Sichtweise führen geschlechtsspezifische Neigungen und Präferenzen $\mathrm{zu}$ einer Selbstselektion der Männer und Frauen in geschlechtstypische Berufsfelder. Innerhalb der neoklassischen Theorie bieten die Humankapitaltheorie und die neue Haushaltsökonomie einen Erklärungsansatz für Geschlechterunterschiede in den Arbeitswerten (Becker 1981, 1964). Die neue Haushaltsökonomie fasst den Haushalt als eine Produktionsgemeinschaft auf, deren Mitglieder innerhäusliche Hausarbeit und außerhäusliche Erwerbsarbeit unter sich aufteilen müssen. Zentral ist hier die Annahme einer geschlechtsspezifischen Arbeitsteilung, bei der zumeist die Frau Hausarbeit und Kinderbetreuung übernimmt, während der Mann sich auf die Erwerbsarbeit konzentriert. Infolge dieser Art der Arbeitsteilung unterscheiden sich Frauen und Männer bezüglich der Kontinuität ihrer Erwerbsarbeit; im Vergleich zu Vätern verzeichnen Mütter häufig Erwerbsunterbrechungen oder Beschäftigungszeiten auf Teilzeitarbeitsplätzen. Da das Humankapital in beiden Fällen nicht oder nur teilweise genutzt wird und Entwertungsprozessen unterliegt, wird erwartet, dass Frauen vergleichsweise geringe Humankapitalinvestitionen tätigen bzw. in solche Qualifikationen investieren, die im Falle einer Erwerbsunterbrechung in nur geringem Maße entwertet werden. Infolge dieser Dequalifikationsgefahren entwickeln Frauen - so die These - andere Arbeitswerte als Männer, die in der Regel eine kontinuierliche Erwerbskarriere anstreben. Insbesondere lässt dieser Erklärungsansatz erwarten, dass Frauen extrinsischen Aspekten wie dem Einkommen und den Aufstiegschancen eine geringere Bedeutung beimessen, dafür Jobeigenschaften, die eine gute Vereinbarung von Erwerbsarbeit und Familie gewährleisten, vergleichsweise hoch schätzen. Die neue Haushaltsökonomie liefert einen konsistenten Erklärungsansatz für die Existenz von beruflicher Segregation, jedoch konnten einige zentrale Annahmen dieses Ansatzes empirisch nicht bestätigt werden. Zum einen zeigt sich, dass Frauen in männertypischen Jobs keine größeren Einkommenseinbußen infolge von Erwerbsunterbrechungen erfahren als Frauen in frauentypischen Berufen (England 1982). Auch die Annahme, dass Frauenberufe eine bessere Vereinbarkeit von Familie und Beruf gewährleisten, hält einer empirischen Überprüfung nicht stand. Im Gegenteil, frauentypische Berufe sind im Vergleich zu Männerberufen durch eine rigidere Arbeitszeitregelung und eine höhere Arbeitsbelastung gekennzeichnet (Glass 1990).

Während ökonomische Ansätze Differenzen in den Arbeitswerten zwischen Männern und Frauen auf Unterschiede im jeweiligen Kosten-Nutzen-Kalkül zurückführen, verortet der sozialisationsorientierte Ansatz die Ursachen geschlechtsspezifischer Arbeitswerte in den unterschiedlichen Sozialisationserfahrungen. Nach diesem Erklärungsansatz entwickeln Mädchen und Jungen im Zuge der Sozialisation stereotype weibliche bzw. männliche Präferenzen und Werte, die wiederum den späteren Berufswunsch prägen und dazu führen, dass vor allem solche Qualifikationen erworben werden, die dem vorherrschenden Geschlechterverständnis entsprechen. Gemäß Reskin (1993) trägt die geschlechtsspezifische Sozialisation auf vier Wegen zur Persistenz von beruflicher Segregation bei, indem a) eine Disposition für geschlechtstypische bzw. eine Zurückweisung von geschlechtsatypischen Berufstätigkeiten gefördert wird, b) eine Neigung zu geschlechtstypischen Arbeitsbedingungen bzw. eine Abneigung von geschlechtsatypischen Arbeitsbedingungen unterstützt wird, c) lediglich Qualifikationen für geschlechtstypische Berufe vermittelt werden, d) vornehmlich Informationen über geschlechtstypische Berufe bereitgestellt werden. Für diesen sozialisationsbasierten Erklärungsansatz findet sich einige empirische Evidenz. Mehrere Studien zeigen, dass die eigene Berufswahl durch die Geschlechtsspezifität des Berufs der Eltern geprägt wird. So ergreifen Personen, deren gleichgeschlechtliches Elternteil eine geschlechtstypische Tätigkeit ausübt, ebenfalls überproportional häufig geschlechtstypische Berufe (Corcoran u. Courant 1987; Dryler 1998; Korupp et al. 2002), wogegen Erwerbstätige, deren Eltern einer gegengeschlechtlichen Tätigkeit nachgehen, selbst häufiger geschlechtsatypische Berufe praktizieren (Okamoto u. England 1999; Waite u. Berryman 1985).

Der angebotsseitige Erklärungsansatz von beruflicher Segregation beruht somit auf zwei zentralen Annahmen. Erstens postuliert dieser Ansatz, dass die individuelle Berufswahl entscheidend durch die Arbeitswerte geprägt wird. Insofern männertypische Berufe eher extrinsische und frauentypische Berufe verstärkt altruistische Gratifikationen bieten (Reskin 1993), ist zu erwarten, dass eine hohe Wertschätzung extrinsischer Arbeitsaspekte mit der Ausübung einer männertypischen Berufstätigkeit korreliert, und eine altruistische Wertorientierung mit einer frauentypischen Tätigkeit assoziiert ist. In den nachfolgenden empirischen Analysen wird daher in einem ersten Schritt die Annahme geprüft, dass Erwerbstätige mit extrinsischen bzw. altruistischen Arbeitswerten verstärkt männer- bzw. frauentypische Tätigkeiten ausüben (Hypothese 1). 
Zweitens basiert der angebotsseitige Erklärungsansatz auf der Annahme von Geschlechterunterschieden in den Arbeitswerten. Diese sollten sich zum einen in geschlechtsspezifischen Wertorientierungen zeigen, indem Männer mehr Wert auf extrinsische Arbeitsaspekte legen als Frauen, wogegen Frauen intrinsischen und altruistischen Aspekten eine höhere Bedeutung beimessen als Männer (Hypothese 2).

Ferner kann angenommen werden, dass sich die Geschlechterunterschiede in den Arbeitswerten infolge des Wandels der Geschlechtervorstellungen als auch der verbesserten Erwerbschancen von Frauen über die Jahre hinweg verringert haben. So haben sich das weibliche als auch das männliche Rollenverständnis während der vergangenen Jahrzehnte deutlich gewandelt (siehe etwa Lee et al. 2007), wodurch in der Sozialisation zusehends modernere Einstellungen zur Frauenerwerbstätigkeit als auch zeitgemäßere Ansichten über eine gerechte Arbeitsteilung zwischen Frauen und Männern vermittelt werden. Zum anderen dürften extrinsische Arbeitsaspekte bei Frauen aufgrund verbesserter Erwerbschancen zusehends an Bedeutung gewinnen.

Die Analyse der Berufswünsche von Aisenbrey u. Brückner (2008) legt in der Tat nahe, dass sich die Berufsvorstellungen von Frauen und Männern im Zeitverlauf angenähert haben. Zwar konstatieren Aisenbrey und Brückner auch für jüngere Kohorten noch immer deutliche Geschlechterunterschiede in den beruflichen Wunschvorstellungen, jedoch hat sich der Anteil an Frauen, der einen geschlechtstypischen Beruf anstrebt, in den vergangenen fünf Jahrzehnten deutlich verringert. Somit ist zu erwarten, dass sich die Geschlechterunterschiede bezüglich der Arbeitswerte im Zeitverlauf verringern, indem sich die Arbeitswerte der Frauen denen der Männer angleichen (Hypothese 3).

Schließlich ist zu erwarten, dass Frauen und Männer sich ebenfalls in der Rangordnung der Arbeitsaspekte unterscheiden. Hier ist anzunehmen, dass Männer extrinsische Jobeigenschaften für wichtiger erachten als intrinsische und altruistische, während Frauen intrinsische und altruistische Aspekte höher schätzen als extrinsische (Hypothese 4).

\section{Daten und Methode}

\subsection{Datenbasis und Variablen}

Für die Analysen werden Daten der „Allgemeinen Bevölkerungsumfrage der Sozialwissenschaften“ (ALLBUS) herangezogen (vgl. Terwey 2000). Die hier zu untersuchenden Arbeitswerte wurden in fünf ALLBUS-Befragungen (1980, 1982, 1991, 1992 und 2000) erhoben. Um mögliche Veränderungen über die Zeit besser abbilden zu können, werden die Daten der Erhebungsjahre 1980 und 1982 sowie 1991 und 1992 in den empirischen Analysen zusammen- gefasst. Da sich die Befragung bis einschließlich 1990 auf deutsche Staatsangehörige beschränkt, werden in den folgenden Analysen nur Personen deutscher Nationalität, die in den alten Bundesländern leben, berücksichtigt. Ferner werden nur Personen zwischen 20 und 60 Jahren in die Analyse aufgenommen. Schließlich beschränkt sich die Analyse auf erwerbstätige Personen. Nichterwerbstätige Personen bleiben unberücksichtigt, da zum einen die Ansprüche an die Erwerbsarbeit zwischen Erwerbstätigen und Nichterwerbstätigen systematisch differieren dürften, und zum anderen, weil Unklarheit herrscht, ob nichterwerbstätige Personen die Wichtigkeit der Arbeitsaspekte auf eine eigene mögliche Erwerbstätigkeit oder aber auf die Erwerbsarbeit im Allgemeinen beziehen. Nach Anwendung dieser Selektionskriterien verbleiben für die Analyse Angaben von 5.150 Personen, von denen 2.090 in den Jahren 1980/82, 1.905 Personen in den Jahren 1991/92 und 1.155 Personen im Jahr 2000 befragt wurden.

Die Arbeitswerte werden im ALLBUS mit folgender Frage erhoben: „Für wie wichtig halten Sie persönlich [folgende Merkmale] für die berufliche Arbeit und den Beruf?“ In den hier berücksichtigten Erhebungsjahren wurde die Wichtigkeit von fünf Jobcharakteristika erfragt: hohes Einkommen, gute Aufstiegschancen im Beruf, interessante Tätigkeit, selbstständige Tätigkeit und helfender Charakter des Berufs. Die Ansprüche an das Einkommen und die Aufstiegschancen lassen sich als extrinsische Arbeitswerte interpretieren, wogegen die Wichtigkeit der übrigen Arbeitsplatzmerkmale intrinsische Arbeitswerte (interessante Tätigkeit, selbständige Tätigkeit) bzw. altruistische Werte (helfender Charakter) darstellen. Die Wichtigkeit der einzelnen Jobcharakteristika wurden jeweils auf einer Likertskala von $1=$ unwichtig bis $7=\mathrm{sehr}$ wichtig angegeben. Als Kovariaten werden in den multivariaten Analysen Alter, Geschlecht, Ausbildungsniveau (kein Abschluss, nicht-akademische Berufsausbildung, Hochschulabschluss), Nettoerwerbseinkommen der Befragungsperson, summiertes Nettoeinkommen aller weiteren Haushaltsmitglieder ${ }^{3}$ (jeweils deflationiert zum Basisjahr 2000), Erwerbsstatus (vollzeitbeschäftigt, teilzeitbeschäftigt, geringfügig erwerbstätig) Familienstand (allein stehend vs. verheiratet oder in nichtehelicher Partnerschaft lebend), das Vorhandensein von Kindern im Haushalt sowie das Erhebungsjahr (1980/82, 1991/92, 2000) berücksichtigt.

Die hier betrachteten Erhebungsjahre unterscheiden sich zum Teil gravierend bezüglich der Wirtschafts- und Ar-

\footnotetext{
${ }^{3}$ Aufgrund vieler fehlender Angaben in den Einkommensvariablen wird ferner jeweils eine Dummy-Variable für fehlende Werte in die Analysen aufgenommen. Für 18\% der Respondenten liegen keine Angaben zum Nettoerwerbseinkommen vor. Ältere Respondenten sowie geringfügig Beschäftige weisen überproportional häufig fehlende Werte in der Einkommensvariable auf. Über die Befragungsjahre hinweg ist der Anteil der fehlenden Werte konstant.
} 
beitsmarktlage. Die frühen 1980er-Jahre waren durch einen starken Wirtschaftsabschwung geprägt, der von steigenden Arbeitslosenzahlen begleitet wurde. In den frühen 1990erJahren setzte eine konjunkturelle Aufschwungphase ein, wodurch die Arbeitslosenquote stark sank. Das Jahr 2000 war ebenfalls durch ein starkes Wirtschaftswachstum und eine abnehmende Arbeitslosenquote gekennzeichnet. Um die unterschiedlichen wirtschaftlichen Rahmenbedingungen in den verschiedenen Erhebungsjahren zu berücksichtigen, wird die aktuelle Arbeitslosenquote auf Bundeslandebene sowie das Wirtschaftswachstum im Befragungsjahr in die multivariaten Analysen aufgenommen.

\subsection{Analysestrategie}

Die empirische Auswertung gliedert sich in drei $\mathrm{Ab}$ schnitte. Zunächst wird der Zusammenhang zwischen den individuellen Arbeitswerten und der Geschlechtstypik des ausgeübten Berufs untersucht. Hiermit wird die oben formulierte Annahme geprüft, wonach Personen mit extrinsischen bzw. altruistischen Arbeitswerten verstärkt männer- bzw. frauentypische Tätigkeiten ausüben (Hypothese 1). Die abhängige Variable in diesem Analyseschritt ist die Geschlechtstypik des ausgeübten Berufs, wobei zwischen Frauenberufen, Männerberufen und Mischberufen unterschieden wird. In Anlehnung an Trappe (2006) werden solche Berufe als frauen- bzw. männertypische Tätigkeiten klassifiziert, in denen der Anteil an weiblichen bzw. männlichen Beschäftigten mehr als $70 \%$ beträgt. ${ }^{4}$ Als zentrale Kovariaten werden die Arbeitswerte berücksichtigt, die in metrischer Form in die Analyse eingehen.

Der zweite Analyseschritt gibt einen deskriptiven Überblick der beruflichen Wertorientierung, indem jeweils für die drei Beobachtungszeitpunkte der Anteil der Personen, der die oben genannten Arbeitswerte für wichtig bzw. sehr wichtig hält, differenziert nach Geschlecht und Altersgruppe ausgewiesen wird. Im dritten, multivariaten Analyseschritt werden zunächst mithilfe von Ordered-Logit-Regressionen (Long 1997) die Arbeitswerte auf die oben beschriebenen sozio-demografischen Merkmale regressiert. Von Interesse ist hier insbesondere, inwiefern sich die Arbeitswerte von Frauen und Männern signifikant voneinander unterscheiden (Hypothese 2). Um zu prüfen, ob sich die Geschlechterunterschiede in den Arbeitswerten im Zeitverlauf verringert haben (Hypothese 3), werden in einem weiteren Schritt die Ordered-Logit-Regressionen getrennt für die drei

\footnotetext{
${ }^{4}$ Der Frauenanteil in den einzelnen Berufen wurde dem vom Institut für Arbeitsmarkt- und Berufsforschung (IAB) herausgegebenen Nachschlagewerk „Berufe im Spiegel der Statistik“ entnommen und auf der Ebene der 3-stelligen „Klassifikation der Berufe“ der Bundesagentur für Arbeit den ALLBUS-Daten zugewiesen. Es werden die vom IAB bereitgestellten Informationen der Jahre 1980, 1991 und 1999 genutzt (Biersack et al. 2000; Parmentier et al. 1993).
}

Erhebungszeiträume geschätzt. Schließlich wird anhand des „Exploded Logit Model for Ranking Data“ untersucht, ob sich die Werteprioritäten von Frauen und Männern unterscheiden (Hypothese 4). Dieses Verfahren wurde zur Analyse von Wahlentscheidungen entwickelt (Allison u. Christakis 1994), jedoch findet es mittlerweile in anderen Bereichen wie der Werteforschung (Klein u. Arzheimer 1999) als auch in der Analyse von Arbeitswerten (Halaby 2003) Anwendung. Für diesen Analyseschritt werden die vorliegenden Rating-Angaben in ein Ranking-Format transformiert, wobei eine gleiche Rangposition mehrerer Arbeitswerte (Rangplatzbindung) möglich ist. ${ }^{5}$ Die abhängige Variable ist somit die ordinale Bewertung der verschiedenen Arbeitsgratifikationen. Da Anwendungen dieses Modells in der Arbeitsmarktforschung bislang eher selten sind, wird es im Folgenden kurz vorgestellt.

Es wird angenommen, dass jede Person $i$ den betrachteten Jobcharakteristika entsprechend des unbeobachteten Nutzens $U_{i j}$, den Person $i$ der Jobeigenschaft $j$ zuordnet, einen bestimmten Rang zuweist. Jeder Nutzenterm $U_{i j}$ besteht aus einer systematischen Komponente $u_{i j}$, die angibt, in welchem Ausmaß die Person $i$ Jobeigenschaft $j$ gegenüber den anderen Jobeigenschaften bevorzugt, und einem Fehlerterm $e_{i j}$. Für zwei Jobeigenschaften $j$ und $k$ beträgt die Wahrscheinlichkeit, dass Jobeigenschaft $j$ einen höheren Nutzen generiert als Jobeigenschaft $k$ somit $P\left(U_{i j}>U_{i k}\right)=\exp \left(u_{i j}\right) /\left(\exp \left(u_{i j}\right)+\exp \left(u_{i k}\right)\right)$. Die Wahrscheinlichkeit, dass Jobeigenschaft $j$ ein höherer Rang als Jobeigenschaft $k$ zugewiesen wird, beträgt damit $\exp \left(u_{i j}-u_{i k}\right)$. Anhand eines Regressionsmodells wird die systematische Komponente $u_{i j}$ geschätzt $\left(u_{i j}=\beta_{j} x_{i}+\gamma z_{j}+\right.$ $\theta w_{i j}$ ), wobei $x_{i}$ die Kovariaten darstellen, die zwischen den Respondenten variieren. Einer der betrachteten Arbeitswerte muss als Referenzkategorie gewählt werden, wodurch $\exp \left(\beta_{j}\right)$ die relative Chancen (Odds Ratios) angibt, dass die Jobeigenschaft $j$ gegenüber der als Referenzkategorie gewählten Jobeigenschaft bevorzugt wird.

\section{Empirische Befunde}

\subsection{Der Zusammenhang zwischen Arbeitswerten und der Geschlechtstypik des Berufs}

Zunächst wird mithilfe einer multinomialen logistischen Regression untersucht, ob zwischen den individuellen

\footnotetext{
${ }^{5} \mathrm{Da}$ es sich bei den zu analysierenden Daten nicht um genuine RankingAngaben handelt, sondern um umgewandelte Rating-Angaben, ist nicht auszuschließen, dass eine Erfassung der betrachteten Werte als Rankings zu abweichenden Ergebnissen führen könnte. Mehrere Studien haben jedoch gezeigt, dass genuine Rankings und in Rankings transformierte Ratings im intra-individuellen Vergleich nahezu identische Präferenzordnungen aufzeigen (Rankin u. Grube 1980; Russel u. Gray 1994).
} 
Tabelle 1 Determinanten der Geschlechtstypik des ausgeübten Berufs (multinomiale logistische Regression, diskrete Effekte in eckigen Klammern)

\begin{tabular}{lllll}
\hline & \multicolumn{2}{c}{ Frauen } & \multicolumn{2}{c}{ Männer } \\
& Frauenberuf & Männerberuf & Frauenberuf & Männerberuf \\
\hline Extrinsische & 0,0694 & $0,1675^{+}$ & 0,0813 & $0,1344^{*}$ \\
Arbeitswerte & {$[-0,0003]$} & {$[0,0158]$} & {$[-0,0023]$} & {$[0,0239]$} \\
Intrinsische & $-0,0709$ & 0,0927 & $-0,0228$ & 0,0465 \\
Arbeitswerte & {$[-0,0260]$} & {$[0,0196]$} & {$[-0,0063]$} & {$[0,0124]$} \\
Altruistische & $0,1446^{* *}$ & $-0,0106$ & $-0,0008$ & $-0,1052^{* *}$ \\
Arbeitswerte & {$[0,0352]$} & {$[-0,0159]$} & {$[0,0085]$} & {$[-0,0238]$} \\
\hline
\end{tabular}

Signifikanzniveau: ${ }^{* *}=p<0,01 ;^{*}=p<0,05 ;^{+}=p<0,1$.

Fallzahl: $\quad 1134 \quad 2007$

LR $\mathrm{Chi}^{2}$-Test $(\mathrm{DF}=22)$ : $\quad 129^{* *} \quad 168^{* *}$

Pseudo-R ${ }^{2}$ : $\quad 0,108 \quad 0,081$

Frauenberuf, Männerberuf: Anteil der weiblichen bzw. männlichen Erwerbstätigen in diesem Beruf mindestens $70 \%$.

Kontrolliert wird für: Alter, Qualifikationsniveau, Ehe- oder Lebenspartner, Kinder im Haushalt, Befragungsjahr.

Quelle: ALLBUS 1980, 1982, 1991, 1992, 2000, eigene Berechnungen
Arbeitswerten und der Ausübung einer frauen- oder männertypischen Beschäftigung ein Zusammenhang besteht (Hypothese 1). In einem ersten Analyseschritt wurden die verschiedenen Arbeitswerte schrittweise in das Modell aufgenommen (ohne Ergebnisdarstellung). Diese Ergebnisse deuteten auf ernstzunehmende Multikollinearitätsprobleme hin, welche auf hohe Korrelationen innerhalb der extrinsischen sowie intrinsischen Arbeitswerte zurückzuführen sind. Zur Vermeidung von Multikollinearität wurden die Angaben zur Einkommenshöhe und den Aufstiegschancen einerseits (extrinsische Werte) sowie zur Ausübung einer interessante Tätigkeit und einer selbständigen Tätigkeit anderseits (intrinsische Werte) anhand von Indizes (ungewichtete Mittelwerte) zusammengefasst. ${ }^{6}$ Die theoretisch hergeleitete Indexbildung wird durch die Ergebnisse einer Faktorenanalyse empirisch unterstützt. Anhand einer Hauptkomponentenanalyse mit anschließender Varimax-Rotation wurden drei Faktoren extrahiert, wobei Faktor 3 jedoch nur einen Eigenwert von 0,79 aufweist. Auf Faktor 1 lassen sich die intrinsischen Arbeitswerte zuordnen (Faktorladungen größer 0,85) und auf Faktor 2 die intrinsischen Werte (Faktorladungen größer 0,83). Faktor 3 ist durch eine hohe Ladung der Variablen „Wichtigkeit helfender Charakter“ gekennzeichnet (die Faktorladung beträgt 0,98).

Die in Tabelle 1 ausgewiesenen Ergebnisse zeigen - in Übereinstimmung mit Hypothese 1 - einen positiven $\mathrm{Zu}$ sammenhang zwischen einer extrinsischen Werthaltung und der Ausübung eines männertypischen Berufs. Ferner scheinen Frauen mit einer altruistischen Werthaltung signifikant häufiger frauentypische Berufe zu ergreifen, während Män-

\footnotetext{
${ }^{6}$ Cronbachs Alpha beträgt 0,70 (extrinsische Arbeitswerte) bzw. 0,71 (in-
} trinsische Arbeitswerte). ner mit einer solchen Wertorientierung die Ausübung männertypischer Tätigkeiten offenbar vermeiden. Obwohl der Zusammenhang zwischen den Arbeitswerten und der Geschlechtstypik des ausgeübten Berufs statistisch signifikant ausfällt, ist die Effektstärke - wie die ausgewiesenen diskreten Effekte ${ }^{7}$ zeigen - als eher gering einzuschätzen. So liegt die Wahrscheinlichkeit, einen männertypischen Beruf auszuüben, bei männlichen Erwerbstätigen, die das Einkommen für sehr wichtig erachten, lediglich um 14 Prozentpunkte $(=6 \times 0,0239)$ über dem Wert der Personen, die die Einkommenshöhe für unwichtig halten. Bei weiblichen Erwerbstätigen beträgt die entsprechende Differenz 9 Prozentpunkte $(=6 \times 0,0158)$. Damit fällt der Effekt der extrinsischen Arbeitswerte bezüglich der Ausübung einer männertypischen Tätigkeit bei weiblichen Erwerbstätigen deutlich niedriger aus als bei männlichen. Dies deutet darauf hin, dass Frauen mit einer hohen extrinsischen Wertorientierung seltener in Männerberufen tätig sind als Männer mit einer vergleichbaren Werthaltung.

4.2 Geschlechterunterschiede in den Arbeitswerten: deskriptive Befunde

In Tabelle 2 wird die Wichtigkeit der einzelnen Arbeitsaspekte, differenziert nach Erhebungszeitpunkt, Geschlecht und Altersgruppe, wiedergegeben. Es lassen sich vier zentrale Befunde festhalten. Erstens zeigt sich, dass intrinsische Arbeitsaspekte, wie die Möglichkeit, eine interessante Tätigkeit auszuüben oder selbstständig arbeiten zu können, für deutlich wichtiger erachtet werden als extrinsische Aspekte

\footnotetext{
${ }^{7}$ Der diskrete Effekt (discrete effect) gibt die Veränderung der geschätzten Wahrscheinlichkeit des Eintreffens eines Ereignisses an, wenn sich die betrachtete unabhängige Variable um eine Einheit erhöht (Long 1997).
} 


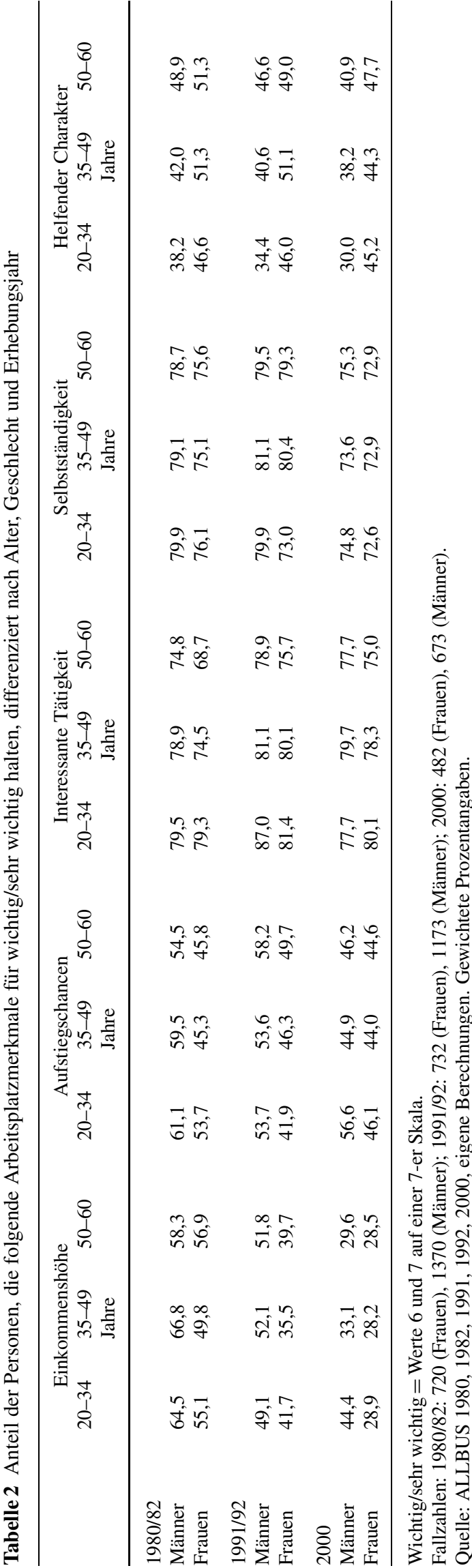

wie ein hohes Einkommen oder gute Aufstiegschancen. Zu allen drei Erhebungszeitpunkten halten mindestens zwei Drittel der befragten Frauen und Männer die Ausübung einer interessanten Tätigkeit und eine autonome Arbeitsweise für wichtig bzw. sehr wichtig, wogegen im Durchschnitt nur etwa jeder zweite Befragte den Verdienstmöglichkeiten und Aufstiegschancen eine hohe Relevanz beimisst. Von den hier betrachteten Arbeitswerten kommt dem helfenden Charakter einer Tätigkeit die geringste Bedeutung zu.

Zweitens ist zu beobachten, dass extrinsische Arbeitsaspekte im Zeitverlauf an Bedeutung verloren haben. Dies gilt insbesondere für das Einkommen. Während in den Jahren 1980/82 zwei Drittel der Männer und die Hälfte der Frauen im mittleren Alter der Höhe des Einkommens eine hohe Relevanz beigemessen haben, fand im Jahr 2000 nur jeder dritte Mann sowie etwa jede vierte Frau die Einkommenshöhe ,wichtig“ bzw. ,sehr wichtig“. Mit Ausnahme der Männer unter 35 Jahren wird im Jahr 2000 die Einkommenshöhe von den hier betrachteten Arbeitsplatzaspekten als am wenigsten wichtig angesehen. Auch haben die Aufstiegschancen sowie der helfende Charakter einer Tätigkeit während des Untersuchungszeitraums an subjektiver Relevanz eingebüßt, wogegen die Wichtigkeit einer interessanten bzw. selbstständigen Tätigkeit im Zeitverlauf relativ stabil geblieben ist.

Für den überraschend starken Bedeutungsrückgang der Einkommenshöhe sind verschiedene Erklärungen denkbar. Eine mögliche Ursache ist in einem allgemeinen Wertewandel zu sehen, infolge dessen materielle Werte generell an Bedeutung verlieren. Empirische Analysen, die eine Verbreitung postmaterieller Werte in den vergangenen Jahrzehnten konstatieren (Hadjar 2006), unterstützen diesen Erklärungsversuch. Eine weitere Ursache kann in Kompositionseffekten infolge der Bildungsexpansion liegen: Wie frühere Studien zeigen, werden materielle Arbeitswerte von höheren Bildungsgruppen für weniger wichtig erachtet als von geringer qualifizierten Personen (Braun u. Borg 2004). Der Bedeutungsrückgang der Einkommenshöhe ist daher möglicherweise auf die im Zeitverlauf prozentuale Zunahme an hochqualifizierten Personen - die dem Einkommen einen eher geringen Stellenwert beimessen zurückzuführen. Als weitere mögliche Ursache kommen Veränderungen der wirtschaftlichen Rahmenbedingungen infrage, die zu Verschiebungen in der Gewichtung der einzelnen Arbeitsaspekte führen können.

Drittens zeigen sich die erwarteten Geschlechterunterschiede in der Bewertung der Arbeitsaspekte. Insgesamt ist zu beobachten, dass Männer eine stärkere extrinsische Orientierung aufweisen als Frauen. Die Einkommenshöhe und die Aufstiegschancen werden zu allen drei Erhebungszeitpunkten und in allen Altersgruppen von Männern für wichtiger erachtet als von Frauen. Hinsichtlich der Bewertung der intrinsischen Arbeitsaspekte zeigen Frauen und 
Männer eine hohe Übereinstimmung, jedoch legen Frauen mehr Wert auf die Ausübung einer Tätigkeit mit helfendem Charakter als Männer. Geschlechterunterschiede in der Präferenzordnung der Arbeitsaspekte sind nicht erkennbar: Sowohl Frauen als auch Männer weisen intrinsischen Aspekten eine höhere Bedeutung zu als den extrinsischen, die wiederum für wichtiger erachtet werden als altruistische Aspekte.

Viertens ist zu erkennen, dass sich Frauen und Männer über den betrachteten Zeitraum hinsichtlich der extrinsischen Arbeitsaspekte teilweise annähern. Dies gilt für Personen der mittleren und oberen Altersgruppe; hier sind im Jahr 2000 nur noch geringe Geschlechterunterschiede in der Bewertung der Höhe des Einkommens und der Aufstiegschancen beobachtbar. Da die Bedeutung der Einkommenshöhe und der Aufstiegschancen bei jungen Frauen stärker zurückgegangen ist als bei jungen Männern, hat sich der Geschlechterunterschied in der jüngsten Altergruppe im Zeitverlauf vergrößert. Tolbert u. Moen (1998), die unter jungen US-amerikanischen Frauen und Männern ebenfalls eine zunehmende Diskrepanz in der Bewertung des Einkommens beobachten, führen diesen Befund darauf zurück, dass sich die Arbeitsmarktchancen der Männer im Vergleich zu denen der Frauen in den vergangenen Jahren deutlich verschlechtert haben. Infolge dieser Entwicklung gewinnen extrinsische Arbeitsaspekte unter männlichen Berufseinsteigern - relativ gesehen - an Bedeutung.

\subsection{Geschlechterunterschiede in den Arbeitswerten:} multivariate Befunde

Im nächsten Schritt werden die Geschlechterunterschiede in den Arbeitswerten anhand der multivariaten OrderedLogit-Regression untersucht. Wie in Tabelle 3 zu erkennen ist, bleiben die Unterschiede zwischen Frauen und Männern auch bei Kontrolle verschiedener sozio-demografischer Merkmale erhalten. So räumen Männer dem Einkommen und den Aufstiegschancen einen signifikant höheren Stellenwert ein als Frauen, wogegen Frauen den helfenden Charakter einer Tätigkeit höher schätzen. Hypothese 2 wird damit bestätigt.

Ferner zeigt sich ein negativer Zusammenhang zwischen dem Lebensalter und der Wichtigkeit extrinsischer sowie intrinsischer Arbeitsaspekte. Dieser Befund entspricht den Ergebnissen früherer Studien (Kalleberg u. Loscocco 1983; Tolbert u. Moen 1998; Wright u. Hamilton 1978). Den Bedeutungsrückgang von extrinsischen Arbeitswerten im Berufsverlauf führen Tolbert u. Moen (1998) primär auf altersbasierte Gratifikations- und Entlohnungsstrukturen zurück. Infolge solcher Entgeltsysteme erhalten jüngere Arbeitnehmer einen verhältnismäßig geringen Lohn, erfahren aber überdurchschnittlich hohe Aufstiegsmöglichkeiten. Da Erwerbstätige insbesondere solche Arbeitsaspekte für wichtig halten, die sie mit hoher Wahrscheinlichkeit auch erreichen können, erwarten Tolbert u. Moen (1998), dass die Wertschätzung extrinsischer Aspekte zum Zeitpunkt des Berufeinstiegs besonders hoch ist und im Berufsverlauf abnimmt. Die geringe Bedeutung von intrinsischen Aspekten in der späten Berufsphase erklären einschlägige Studien häufig anhand der Disengagement-Theorie (siehe Kalleberg u. Loscocco 1983; Lorence 1987). Hier wird angenommen, dass sich ältere Menschen aus der Gesellschaft zurückziehen und damit auch der Erwerbstätigkeit eine eher geringe Relevanz beimessen. Da die vorliegenden Untersuchungen nicht auf Längsschnittdaten basieren, bleibt jedoch letztendlich ungewiss, inwiefern die hier beobachtete Korrelation zwischen dem Lebensalter und den Arbeitswerten tatsächlich auf Alterseffekten oder aber auf Kohorteneffekten beruht.

Einen starken Effekt auf die Wichtigkeit der betrachteten Arbeitsaspekte hat ferner das Bildungsniveau. Hochschulabsolventen messen der Einkommenshöhe und den Aufstiegschancen eine geringere Bedeutung $\mathrm{zu}$ als Geringqualifizierte oder Berufsschulabsolventen, wogegen intrinsische Aspekte wie die Ausübung einer interessanten und selbstbestimmten Tätigkeit von Hochschul- sowie Berufsschulabsolventen höher geschätzt werden als von Personen ohne abgeschlossene Ausbildung. Vermutlich ist die geringere Wertschätzung des Einkommens und der Aufstiegschancen unter den Hochqualifizierten Ausdruck einer stärkeren postmateriellen Werthaltung.

Frauen und Männer in einer festen Partnerschaft oder Ehe halten ihre Karrierechancen für wichtiger als allein lebende Personen. Dieses Ergebnis steht im Einklang mit Gormans (2000) Befunden, wonach Ehepaare dem Berufserfolg eine größere Bedeutung beimessen als ledige Personen, da sich im Zuge der Partnerschaft die Wohn- und Konsumgewohnheiten ändern und sich höhere Ansprüche an den Lebensstandard herausbilden. Überraschenderweise hat das Vorhandensein von Kindern so gut wie keinen signifikanten Effekt auf die untersuchten Arbeitswerte. Bemerkenswert ist insbesondere der nichtsignifikante Effekt von Elternschaft auf die extrinsischen Arbeitswerte. Dieser Befund widerspricht der Annahme, dass Eltern aufgrund der größeren finanziellen Belastung dem Einkommen eine höhere Bedeutung zumessen als kinderlose Personen. Hier ist jedoch denkbar, dass sich gegenläufige Effekte bei Frauen und Männern gegenseitig aufheben. So wurde in früheren Studien oftmals die These formuliert, dass insbesondere Väter eine starke extrinsische Orientierung aufweisen, wogegen Mütter aufgrund ihres häufig geringen beruflichen Engagements extrinsischen Arbeitsaspekten einen niedrigeren Stellenwert beimessen als kinderlose Frauen (De Vaus u. McAllister 1991; Mardsen et al. 1993). Um zu prüfen, ob die familiäre Situation in unterschiedlicher Weise die Arbeitswerte von Frauen und Männern prägt, wurden 
Tabelle 3 Determinanten der Arbeitswerte (Ordered-LogitRegression, Standardfehler in Klammern)

\begin{tabular}{|c|c|c|c|c|c|}
\hline & $\begin{array}{l}\text { Einkommens- } \\
\text { höhe }\end{array}$ & $\begin{array}{l}\text { Aufstiegs- } \\
\text { chancen }\end{array}$ & $\begin{array}{l}\text { Interessante } \\
\text { Tätigkeit }\end{array}$ & $\begin{array}{l}\text { Selbst- } \\
\text { ständigkeit }\end{array}$ & $\begin{array}{l}\text { Helfender } \\
\text { Charakter }\end{array}$ \\
\hline Frau & $\begin{array}{l}-0,2867^{* *} \\
(0,0632)\end{array}$ & $\begin{array}{l}-0,2556^{* *} \\
(0,0625)\end{array}$ & $\begin{array}{l}0,1021 \\
(0,0663)\end{array}$ & $\begin{array}{l}0,0346 \\
(0,0654)\end{array}$ & $\begin{array}{l}0,3897^{* *} \\
(0,0619)\end{array}$ \\
\hline Alter in Jahren & $\begin{array}{l}-0,0071^{* *} \\
(0,0026)\end{array}$ & $\begin{array}{l}-0,0079^{* *} \\
(0,0026)\end{array}$ & $\begin{array}{l}-0,0131^{* *} \\
(0,0027)\end{array}$ & $\begin{array}{l}0,0012 \\
(0,0027)\end{array}$ & $\begin{array}{l}0,0152^{* *} \\
(0,0025)\end{array}$ \\
\hline $\begin{array}{l}\text { Ehe- oder Lebens- } \\
\text { partner }\end{array}$ & $\begin{array}{l}0,1034 \\
(0,0716)\end{array}$ & $\begin{array}{l}0,1925^{* *} \\
(0,0712)\end{array}$ & $\begin{array}{l}0,0531 \\
(0,0749)\end{array}$ & $\begin{array}{l}0,0082 \\
(0,0743)\end{array}$ & $\begin{array}{l}0,0224 \\
(0,0706)\end{array}$ \\
\hline Kind im Haushalt & $\begin{array}{l}0,0719 \\
(0,0570)\end{array}$ & $\begin{array}{l}-0,0562 \\
(0,0566)\end{array}$ & $\begin{array}{l}-0,0355 \\
(0,0599)\end{array}$ & $\begin{array}{l}0,0364 \\
(0,0597)\end{array}$ & $\begin{array}{l}0,1483^{* *} \\
(0,0563)\end{array}$ \\
\hline Berufsausbildung & $\begin{array}{l}-0,0281 \\
(0,0795)\end{array}$ & $\begin{array}{l}0,0268 \\
(0,0789)\end{array}$ & $\begin{array}{l}0,3559^{* *} \\
(0,0811)\end{array}$ & $\begin{array}{l}0,2715^{* *} \\
(0,0813)\end{array}$ & $\begin{array}{l}0,0382 \\
(0,0775)\end{array}$ \\
\hline Hochschulabschluss & $\begin{array}{l}-0,8482^{* *} \\
(0,1012)\end{array}$ & $\begin{array}{l}-0,5887^{* *} \\
(0,1007)\end{array}$ & $\begin{array}{l}0,5439^{* *} \\
(0,1048)\end{array}$ & $\begin{array}{l}0,3358^{* *} \\
(0,1042)\end{array}$ & $\begin{array}{l}-0,0217 \\
(0,0991)\end{array}$ \\
\hline $\begin{array}{l}\text { Nettoerwerbs- } \\
\text { einkommen (log.) }\end{array}$ & $\begin{array}{l}0,1412^{*} \\
(0,0619)\end{array}$ & $\begin{array}{l}0,1402^{*} \\
(0,0613)\end{array}$ & $\begin{array}{l}0,1385^{*} \\
(0,0650)\end{array}$ & $\begin{array}{l}0,2648^{* *} \\
(0,0650)\end{array}$ & $\begin{array}{l}-0,1365^{*} \\
(0,0618)\end{array}$ \\
\hline $\begin{array}{l}\text { Sum. Einkommen anderer } \\
\text { HH.mitglieder (log.) }\end{array}$ & $\begin{array}{l}0,0088 \\
(0,0096)\end{array}$ & $\begin{array}{l}-0,0118 \\
(0,0095)\end{array}$ & $\begin{array}{l}0,0126 \\
(0,0100)\end{array}$ & $\begin{array}{l}0,0256^{* *} \\
(0,0099)\end{array}$ & $\begin{array}{l}0,0099 \\
(0,0094)\end{array}$ \\
\hline Teilzeitbeschäftigt & $\begin{array}{l}-0,2075^{*} \\
(0,0939)\end{array}$ & $\begin{array}{l}0,0210 \\
(0,0928)\end{array}$ & $\begin{array}{c}-0,0836 \\
(0,0975)\end{array}$ & $\begin{array}{l}0,0104 \\
(0,0963)\end{array}$ & $\begin{array}{l}-0,1536^{+} \\
(0,0910)\end{array}$ \\
\hline Geringfügig beschäftigt & $\begin{array}{l}-0,3526^{* *} \\
(0,1210)\end{array}$ & $\begin{array}{l}-0,0555 \\
(0,1211)\end{array}$ & $\begin{array}{l}0,0140 \\
(0,1255)\end{array}$ & $\begin{array}{l}0,0646 \\
(0,1252)\end{array}$ & $\begin{array}{l}-0,2978^{*} \\
(0,1206)\end{array}$ \\
\hline Jahr 1990/91 & $\begin{array}{l}-0,5999^{* *} \\
(0,0945)\end{array}$ & $\begin{array}{l}-0,2212^{*} \\
(0,0933)\end{array}$ & $\begin{array}{l}0,0218 \\
(0,0987)\end{array}$ & $\begin{array}{l}-0,2214^{*} \\
(0,0984)\end{array}$ & $\begin{array}{l}-0,0132 \\
(0,0920)\end{array}$ \\
\hline Jahr 2000 & $\begin{array}{l}-1,0341^{* *} \\
(0,1093)\end{array}$ & $\begin{array}{l}-0,1921^{+} \\
(0,1074)\end{array}$ & $\begin{array}{l}0,0319 \\
(0,1140)\end{array}$ & $\begin{array}{l}-0,3969^{* *} \\
(0,1133)\end{array}$ & $\begin{array}{l}-0,1065 \\
(0,1064)\end{array}$ \\
\hline Arbeitslosenquote & $\begin{array}{l}-0,0316^{*} \\
(0,0126)\end{array}$ & $\begin{array}{l}-0,0318^{*} \\
(0,0125)\end{array}$ & $\begin{array}{c}-0,0059 \\
(0,0133)\end{array}$ & $\begin{array}{c}-0,0049 \\
(0,0132)\end{array}$ & $\begin{array}{l}-0,0277^{*} \\
(0,0123)\end{array}$ \\
\hline Wirtschaftswachstum & $\begin{array}{l}0,0033 \\
(0,0259)\end{array}$ & $\begin{array}{l}0,0029 \\
(0,0257)\end{array}$ & $\begin{array}{l}-0,0299 \\
(0,0272)\end{array}$ & $\begin{array}{l}0,0071 \\
(0,0270)\end{array}$ & $\begin{array}{l}-0,0198 \\
(0,0255)\end{array}$ \\
\hline \multicolumn{6}{|c|}{ Signifikanzniveau: ${ }^{* *}=p<0,01 ;^{*}=p<0,05 ;^{+}=p<0,1$} \\
\hline Fallzahl: & 5148 & 5139 & 5143 & 5144 & 5144 \\
\hline LR Chi ${ }^{2}$-Test $(\mathrm{DF}=16)$ : & $590,16^{* *}$ & $175,69^{* *}$ & $72,74^{* *}$ & $81,02^{* *}$ & $126,43^{* *}$ \\
\hline Pseudo- $\mathrm{R}^{2}$ : & 0,11 & 0,04 & 0,02 & 0,02 & 0,02 \\
\hline \multicolumn{6}{|c|}{$\begin{array}{l}\text { Referenzkategorien: Mann, kein Ehe- oder Lebenspartner, kein Kind im Haushalt, keine abgeschlossene Be- } \\
\text { rufsausbildung, vollzeitbeschäftigt, Befragungszeitpunkt 1982/82. } \\
\text { Im Modell enthalten sind ferner } 2 \text { Dummy-Variablen für fehlende Einkommensangaben. } \\
\text { Quelle: ALLBUS 1980,1982, 1991, 1992, 2000, eigene Berechnungen }\end{array}$} \\
\hline
\end{tabular}

in einer weiteren Modellspezifikation Interaktionsterme zwischen dem Geschlecht einerseits und den Variablen „Lebens- oder Ehepartner“ sowie „Kind im Haushalt“ andererseits berücksichtigt (ohne Ergebnisdarstellung). Der Haupteffekt des Geschlechts bleibt hinsichtlich der extrinsischen und altruistischen Arbeitswerte weitgehend konstant und die Interaktionseffekte sind nicht signifikant. Die in früheren Studien formulierte Annahme, dass Mütter weniger Wert auf extrinsische Gratifikationen legen, wird damit nicht bestätigt. Bezüglich der intrinsischen Werte sind nun deutlich größere Geschlechterkoeffizienten zu beobachten. Der Geschlechterkoeffizient im Modell ,inte- ressante Tätigkeit" beträgt jetzt 0,3108 (T-Wert: 2,96) und im Modell „Selbstständigkeit“ 0,1856 (T-Wert: 1,80). Die Analysen zeigen somit, dass bei partner- und kinderlosen Personen signifikante Geschlechterunterschiede in den intrinsischen Arbeitswerten bestehen, wogegen Männer und Frauen mit einer Familie sich in der Bewertung der intrinsischen Arbeitsaspekte kaum unterscheiden.

Wie schon in den deskriptiven Analysen erkennbar war, zeigen auch die multivariaten Berechnungen einen Rückgang der extrinsischen Arbeitswerte im Zeitverlauf. Im obigen Abschnitt wurden drei Erklärungen für den Bedeutungsverlust der Einkommenshöhe skizziert: der generelle 
Tabelle 4 Effekt des Geschlechts auf die Arbeitswerte (Ordered-Logit-

Regressionen, getrennt für 1980/82, 1991/92 und 2000)

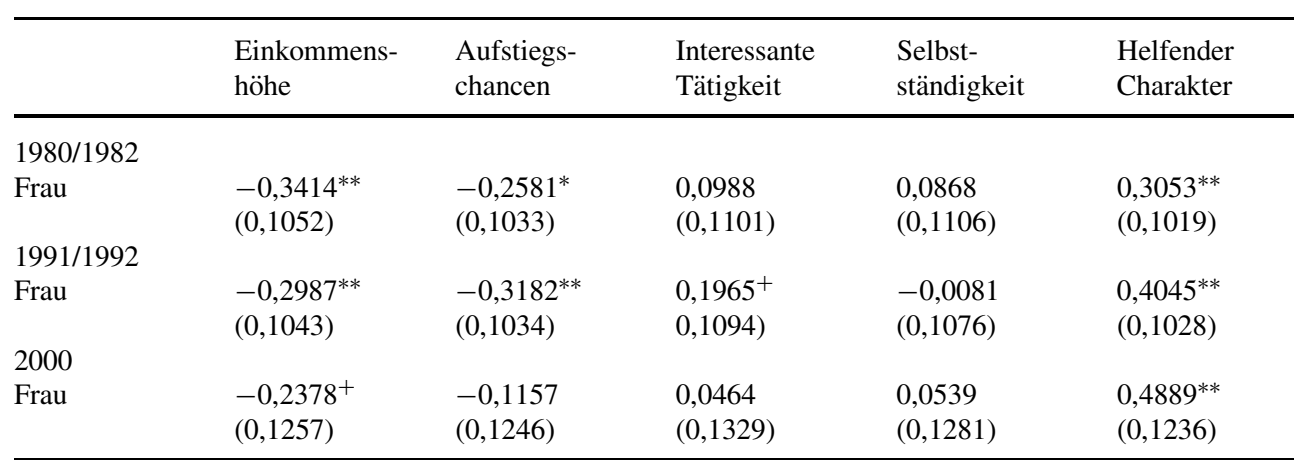

Signifikanzniveau: ${ }^{* *}=p<0,01 ;^{*}=p<0,05 ;^{+}=p<0,1$.

Fallzahlen: 1980/1982: 2049; 1991/92: 1910; 2000: 1185.

Kontrolliert wird für: Alter, Ehe-/Lebenspartner, Kind im Haushalt, Qualifikationsniveau, Nettoerwerbseinkommen, summiertes Nettoeinkommen anderer Haushaltsmitglieder, fehlende Einkommensangaben (2 Dummy-Variablen), Erwerbstatus.

Quelle: ALLBUS 1980, 1982, 1991, 1992, 2000, eigene Berechnungen
Wertewandel, Kompositionseffekte infolge der Bildungsexpansion sowie Effekte der Wirtschaftslage. Da in Tabelle 3 auch bei Kontrolle des Bildungsniveaus sowie der Arbeitsmarkt- und Wirtschaftslage ein signifikanter Bedeutungsrückgang des Einkommens und der Aufstiegschancen zu beobachten ist, gründet die abnehmende Wertschätzung dieser Arbeitsaspekte anscheinend primär in einem generellen Wertewandel.

In einem weiteren Analyseschritt wird die OrderedLogit-Regression jeweils getrennt für die drei Erhebungszeiträume geschätzt. Hiermit werden mögliche Veränderungen der Geschlechterunterschiede in den Arbeitswerten über den hier betrachteten Zeitraum hinweg abgebildet (Hypothese 3). Die Geschlechterkoeffizienten dieser Regressionen sind in Tabelle 4 ausgewiesen.

Wie ersichtlich ist, haben sich die Geschlechterunterschiede in den extrinsischen Arbeitswerten im Zeitverlauf verringert. $\mathrm{Zu}$ Beginn der 1980er-Jahre bestehen signifikante Unterschiede in der Beurteilung des Einkommens und der Aufstiegschancen zwischen Männern und Frauen, bis zum Jahr 2000 haben sich diese Unterschiede jedoch deutlich abgeschwächt und sind nur bezüglich des Einkommens schwach signifikant. Hinsichtlich der intrinsischen Werte sind zu allen drei Zeitpunkten kaum Unterschiede zwischen Männern und Frauen erkennbar, wogegen die Geschlechterunterschiede in den altruistischen Werten im Zeitverlauf zugenommen haben. Hypothese 3, wonach sich die Arbeitswerte von Frauen und Männern im Zeitverlauf angleichen, findet somit partielle empirische Unterstützung.

Im nächsten Analyseschritt wird nun anhand der erstellten Ranking-Angaben untersucht, inwiefern sich Frauen und Männer in ihren Präferenzordnungen der Arbeitsaspekte unterscheiden (Hypothese 4). Als Referenzkategorie dient die Wertschätzung einer, ,interessanten Tätigkeit“.
Auch hier wird die Analyse getrennt für die jeweiligen Erhebungszeitpunkte durchgeführt. Die Geschlechterkoeffizienten der Modellschätzungen sind in Tabelle 5 ausgewiesen. Anhand dieser Koeffizienten werden die relativen Chancen (Odds Ratios) berechnet, dass Frauen im Vergleich zu Männern intrinsische bzw. altruistische Arbeitsaspekte gegenüber extrinsischen bevorzugen.

Wie die in Tabelle 6 ausgewiesenen Odds Ratios zeigen, scheinen Frauen intrinsischen Werten eine höhere Priorität zuzuordnen als Männer. Gemäß unseren Berechnungen sind im Erhebungszeitraum 1980/82 die Chancen, dass Frauen dem Interesse an der Tätigkeit eine höhere Priorität zuweisen als dem Einkommen, um den Faktor 1,46 größer als die entsprechenden Chancen der Männer. ${ }^{8}$ Die Chancen, dass Frauen dem helfenden Charakter einer Tätigkeit eine größere Bedeutung als der Einkommenshöhe beimessen, sind sogar um das 1,71fache größer als die der männlichen Erwerbstätigen. ${ }^{9}$ Analog zum Ergebnismuster in Tabelle 4 zeigt sich, dass die Geschlechterunterschiede im Zeitverlauf stark abnehmen. Während im Zeitraum 1980/82 bei allen ausgewiesenen paarweisen Präferenzen signifikante Unterschiede zwischen Männern und Frauen bestehen, trifft dies im Jahr 2000 lediglich auf die Rangordnung der extrinsischen und altruistischen Arbeitsaspekte zu. Folglich weisen Frauen im Jahr 2000 den extrinsischen gegenüber den intrinsischen Aspekten keine signifikant niedrigere (oder höhere) Priorität zu als Männer. Gemäß den vorliegenden empirischen Befunden bestehen somit aktuell nur noch geringfügige Unterschiede in den Präferenzordnungen von Frauen und Männern.

\footnotetext{
${ }^{8}$ Dieser Odds Ratio wurde anhand der in Tabelle 5 ausgewiesenen Koeffizienten folgendermaßen berechnet: $1 / \exp (-0,3781)=1,46$.

${ }^{9}=1 / \exp (-0,3781-0,1616)=1,71$.
} 
Tabelle 5 Effekt des Geschlechts auf die Arbeitswerte (Exploded Logit Model for Ranking Data)

\begin{tabular}{llllll}
\hline & $\begin{array}{l}\text { Einkommens- } \\
\text { höhe }\end{array}$ & $\begin{array}{l}\text { Aufstiegs- } \\
\text { chancen }\end{array}$ & $\begin{array}{l}\text { Interessante } \\
\text { Tätigkeit }\end{array}$ & $\begin{array}{l}\text { Selbst- } \\
\text { ständigkeit }\end{array}$ & $\begin{array}{l}\text { Helfender } \\
\text { Charakter }\end{array}$ \\
\hline $\begin{array}{l}\text { Cond } \\
\text { Frau }\end{array}$ & & & & & \\
& $-0,3781^{* *}$ & $-0,3249^{*}$ & 0 & $-0,0156$ & 0,1616 \\
$1991 / 1992$ & $(0,1447)$ & $(0,1421)$ & - & $(0,1474)$ & $(0,1471)$ \\
Frau & $-0,3024^{*}$ & $-0,2578^{+}$ & 0 & 0,2000 & $0,2560^{+}$ \\
2000 & $(0,1476)$ & $(0,1460)$ & - & $(0,1501)$ & $(0,1513)$ \\
Frau & $-0,1079$ & $-0,0951$ & 0 & $-0,0876$ & $0,4417^{*}$ \\
& $(0,1861)$ & $(0,1821)$ & - & $(0,1835)$ & $(0,1889)$ \\
\hline
\end{tabular}

Signifikanzniveau: ${ }^{* *}=p<0,01 ;^{*}=p<0,05 ;^{+}=p<0,1$.

Fallzahlen: 1980/82: 2049; 1991/92: 1910; 2000: 1185.

Pseudo-R ${ }^{2}$ : 1980/82: 0,12; 1991/92: 0,17; 2000: 0,18.

Kontrolliert wird für: Alter, Ehe-/Lebenspartner, Kind im Haushalt, Qualifikationsniveau, Nettoerwerbseinkommen, summiertes Nettoeinkommen anderer Haushaltsmitglieder, fehlende Einkommensangaben (2 Dummy-Variablen), Erwerbstatus.

Quelle: ALLBUS 1980, 1982, 1991, 1992, 2000, eigene Berechnungen
Tabelle 6 Odds Ratios des Geschlechtereffekts auf die paarweisen Präferenzen der Arbeitswerte (basierend auf Tabelle 5)

\begin{tabular}{|c|c|c|c|}
\hline & $\begin{array}{l}\text { Interessante } \\
\text { Tätigkeit }\end{array}$ & $\begin{array}{l}\text { Selbst- } \\
\text { ständigkeit }\end{array}$ & $\begin{array}{l}\text { Helfender } \\
\text { Charakter }\end{array}$ \\
\hline \multicolumn{4}{|l|}{$1980 / 82$} \\
\hline Einkommenshöhe & $\begin{array}{l}1,46^{* *} \\
(6,83)\end{array}$ & $\begin{array}{l}1,43^{*} \\
(6,23)\end{array}$ & $\begin{array}{l}1,71^{* *} \\
(16,40)\end{array}$ \\
\hline Aufstiegschancen & $\begin{array}{l}1,38^{*} \\
(5,22)\end{array}$ & $\begin{array}{l}1,36^{*} \\
(4,72)\end{array}$ & $\begin{array}{l}1,63^{* *} \\
(14,45)\end{array}$ \\
\hline \multicolumn{4}{|l|}{$1991 / 92$} \\
\hline Einkommenshöhe & $\begin{array}{l}1,35^{*} \\
(4,20)\end{array}$ & $\begin{array}{l}1,10 \\
(0,52)\end{array}$ & $\begin{array}{l}1,73^{* *} \\
(20,10)\end{array}$ \\
\hline Aufstiegschancen & $\begin{array}{l}1,29^{+} \\
(3,12)\end{array}$ & $\begin{array}{l}1,06 \\
(0,17)\end{array}$ & $\begin{array}{l}1,71^{* *} \\
(17,01)\end{array}$ \\
\hline \multicolumn{4}{|l|}{2000} \\
\hline Einkommenshöhe & $\begin{array}{l}1,11 \\
(0,34)\end{array}$ & $\begin{array}{l}1,02 \\
(0,01)\end{array}$ & $\begin{array}{l}1,73^{* *} \\
(13,40)\end{array}$ \\
\hline Aufstiegschancen & $\begin{array}{l}1,10 \\
(0,27)\end{array}$ & $\begin{array}{l}1,01 \\
(0,00)\end{array}$ & $\begin{array}{l}1,71^{* *} \\
(12,17)\end{array}$ \\
\hline
\end{tabular}

Wald $\chi^{2}$-Werte in Klammern

\section{Schlussfolgerungen}

Verschiedene Erklärungen der beruflichen Segregation basieren auf der Annahme von Geschlechterunterschieden in den Arbeitswerten. Sowohl neoklassische Ansätze als auch die Sozialisationstheorie postulieren, dass Frauen und Männer unterschiedliche Arbeitswerte entwickeln, die sich in einer geschlechtspezifischen Berufswahl äußern und somit zur Reproduktion von beruflicher Segregation beitragen. Mit der vorliegenden Studie wird die Annahme geschlechtsspezifischer Arbeitswerte erstmals für Deutschland ausführlich untersucht.

Die durchgeführten Analysen zeigen einige Geschlechterunterschiede in den Arbeitswerten, allerdings haben sich diese während des betrachteten Zeitraums zum Teil deutlich verringert. Während zu Beginn der 1980er-Jahre noch signifikante Unterschiede bestehen, indem Männer mehr Wert auf gute Verdienstmöglichkeiten sowie hohe Aufstiegschancen legen und Frauen die Ausübung einer Tätigkeit mit helfendem Charakter höher schätzen, sind markante Geschlechterunterschiede im Jahr 2000 nur hinsichtlich der altruistischen Arbeitswerte zu beobachten. Trotz der in den 1980er- und 1990er-Jahren konstatierten Geschlechterunterschiede sind die Divergenzen zwischen Männern und Frauen als eher gering einzuschätzen. Empirische Analysen neigen häufig dazu, anhand signifikanter Koeffizienten vor allem Differenzen hervorzuheben, dabei jedoch mögliche Muster der Übereinstimmung zu übersehen. Wie die deskriptiven Analysen gezeigt haben, ist die grundsätzliche Bewertung der verschiedenen Arbeitsaspekte bei Männern und Frauen trotz einiger Unterschiede in einzelnen Arbeitswerten relativ ähnlich. Beide Geschlechter messen intrinsischen Aspekten wie der Ausübung einer interessanten und selbstbestimmten Tätigkeit eine höhere Bedeutung zu als den Verdienstmöglichkeiten und den Aufstiegschancen.

Anhand der vorliegenden Ergebnisse lässt sich die Leistungsfähigkeit der angebotsseitigen Erklärungsansätze von beruflicher Segregation einschätzen. Drei Befunde der durchgeführten Analysen deuten darauf hin, dass sich Geschlechtersegregation nur bedingt durch die hier diskutierten Ansätze erklären lässt. Zum einen sind die Gemeinsamkeiten zwischen Männern und Frauen bezüglich ihrer Arbeitswerte deutlich größer als die Unterschiede. Dies zeigt sich vor allem in den geringen Unterschieden in den Präferenzordnungen. Zweitens ist hinsichtlich der Arbeitswerte eine starke Angleichung zwischen den Geschlechtern im Zeitverlauf zu beobachten, wogegen das Ausmaß der beruflichen Segregation im gleichen 
Zeitraum so gut wie unverändert geblieben ist (Brückner 2004; Rosenfeld u. Trappe 2002). Somit haben offenbar geschlechtsspezifische Veränderungen in den Arbeitswerten keine Auswirkungen auf Persistenz von beruflicher Segregation. Drittens besteht ein zwar signifikanter, aber nur schwach ausgeprägter Zusammenhang zwischen den individuellen Arbeitswerten und der Geschlechtstypik des ausgeübten Berufs, was darauf hindeutet, dass die Allokation auf frauen- und männertypische Arbeitsplätze nicht primär durch die Arbeitswerte geleitet wird.

Auch die bei Frauen beobachtete höhere Wertschätzung von altruistischen Arbeitsaspekten erklärt nicht die Konzentration von weiblichen Erwerbstätigen in verhältnismäßig gering entlohnten Berufen. Extrinsische und altruistische Arbeitsaspekte schließen sich nicht gegenseitig aus. Die hier aufzeigten Geschlechterunterschiede in den Arbeitswerten erklären nicht, wieso Frauen mit einer Präferenz für helfende Tätigkeiten offenbar eher in Lehrund Pflegeberufen beschäftigt sind, wogegen Männer mit einer ähnlichen Werthaltung anscheinend häufiger besser bezahlte medizinische und juristische Berufe ergreifen.

Insgesamt legen die Befunde dieser Studie nahe, dass die Ursachen von Geschlechtersegregation eher auf der Nachfrageseite des Arbeitsmarktes verortet sind. Hier stellt sich die Frage, in welchem Maße Geschlechterdiskriminierung im Rekrutierungsprozess vorherrscht, die dazu führt, dass sich Frauen bei der Berufswahl infolge geringer Zugangschancen in bestimmte Tätigkeiten auf frauentypische Berufe beschränken. Die meisten empirischen Studien bezüglich der Ursachen von beruflicher Segregation - wie auch diese - betrachten allein die Angebotsseite und blenden aufgrund des schwierigen Datenzugangs die Nachfrageseite des Arbeitsmarkes aus. US-amerikanische Studien erbringen gewisse empirische Evidenz für diskriminierende Einstellungs- und Allokationsentscheidungen der Arbeitgeber (Petersen u. Saporta 2004), für den deutschen Arbeitsmarkt stehen entsprechende Studien noch aus. Der Befund von Aisenbrey u. Brückner (2008), dass es Frauen seltener als Männern gelingt, ihren Berufswunsch zu verwirklichen, kann allerdings als ein Indiz für diskriminierende Einstellungspraktiken im deutschen Arbeitsmarkt gewertet werden.

Des Weiteren liefert der vorliegende Beitrag interessante Erkenntnisse bezüglich der Analyse von Arbeitsgratifikationen. Ein Großteil der Studien, die sich mit arbeitsmarktbezogenen Gratifikationen befassen, betrachtet Unterschiede in der Einkommenshöhe. Wie die vorliegenden Analysen zeigten, werden intrinsische Arbeitsplatzaspekte jedoch von der Mehrheit der Erwerbstätigen für wichtiger erachtet als extrinsische. Dieser Befund legt nahe, dass die Verwendung des Erwerbseinkommens als Gesamtindikator für die Arbeitsgratifikationen, wie häufig in der Arbeitsmarktforschung praktiziert wird, zu kurz greift. Mit ihrer eindimensionalen Fokussierung auf rein monetäre Aspekte vernachlässigen viele Studien schlichtweg, dass Arbeitsgratifikationen mehrdimensional ausgerichtet sind und der Stellenwert der einzelnen Gratifikationen zwischen verschiedenen Personengruppen - etwa Alters- oder Qualifikationsgruppen - variieren kann. So stellen ältere Personen, wie bereits frühere Studien, aber auch die hier durchgeführten Analysen gezeigt haben, andere Ansprüche an die Erwerbsarbeit als Berufseinsteiger. Die vorliegenden Ergebnisse legen damit nahe, die subjektive Relevanz der verschiedenen Arbeitsgratifikationen stärker zu berücksichtigen und von der fast ausschließlichen Fokussierung auf extrinsische Gratifikationen abzurücken.

\section{Kurzfassung}

Eine wesentliche Ursache der Einkommensungleichheit zwischen Frauen und Männern wird in der geschlechtsspezifischen Segregation des Arbeitsmarktes gesehen, infolge deren Frauen oftmals in Berufsfeldern mit vergleichsweise niedriger Entlohnung und geringen Aufstiegschancen beschäftigt sind. Während eine Vielzahl von Studien die Folgen beruflicher Segregation untersucht, kommt deren ursächlichen Faktoren nur wenig Aufmerksamkeit zu. Mögliche Ursachen von geschlechtsspezifischer Segregation und den damit einhergehenden Lohnunterschieden zwischen Männern und Frauen finden sich sowohl auf der Nachfrageals auch Angebotsseite des Arbeitsmarktes. Auf der Nachfrageseite kann berufliche Segregation aus diskriminierenden Einstellungs- und Allokationsentscheidungen der Arbeitgeber resultieren, die dazu führen, dass Frauen auf schlechter bezahlte und statusniedrigere Berufspositionen als gleich qualifizierte Männer platziert werden. Angebotsseitige Erklärungsansätze - hierzu zählen neoklassische Ansätze und Sozialisationstheorien - betrachten dagegen berufliche Segregation als Ausdruck unterschiedlicher Arbeitswerte und -präferenzen. Während der neoklassische Ansatz geschlechtsspezifische Segregation darauf zurückführt, dass Frauen in einer rationalen Entscheidungssituation geringer entlohnte, aber dafür familienkompatible Berufe wählen, sieht der Sozialisationsansatz die primäre Ursache von Arbeitsmarktsegregation in der geschlechtsspezifischen Vermittlung von extrinsischen bzw. altruistischen Wertorientierungen bei Jungen und Mädchen.

Die Frage nach möglichen Geschlechterunterschieden in den Arbeitswerten ist somit zentral für die Analyse der Ursachen von beruflicher Segregation. Insofern gravierende Unterschiede zwischen Männern und Frauen existieren, wären die ungünstigere Einkommenssituation und Berufsstellung der Frauen nicht allein auf diskriminierende Einstellungs- und Beförderungspraktiken der Arbeitgeber zurückzuführen, sondern müssten zumindest partiell als 
Konsequenz des eigenen Berufswahlverhaltens begriffen werden.

Obwohl in angebotsseitigen Erklärungsansätzen der Annahme geschlechtsspezifischer Arbeitswerte eine zentrale Rolle zukommt, wurde sie - zumindest für Deutschland - bislang kaum einer empirischen Prüfung unterzogen. In dem vorliegenden Beitrag wird anhand der Daten des ALLBUS die Struktur und Entwicklung von Geschlechterunterschieden in den Arbeitswerten zwischen 1980 und 2000 untersucht. In den ALLBUS-Erhebungen wurde die Wichtigkeit von fünf Jobcharakteristika erfragt: hohes Einkommen, gute Aufstiegschancen im Beruf, interessante Tätigkeit, selbstständige Tätigkeit und helfender Charakter des Berufs. Die Ansprüche an das Einkommen und die Aufstiegschancen lassen sich als extrinsische Arbeitswerte interpretieren, wogegen die Wichtigkeit der übrigen Arbeitsplatzmerkmale intrinsische Arbeitswerte (interessante Tätigkeit, selbständige Tätigkeit) bzw. altruistische Werte (helfender Charakter) darstellen.

Die empirischen Analysen zeigen, dass Männer mehr Wert auf extrinsische Arbeitsaspekte legen, während Frauen altruistische Aspekte höher schätzen. Über den betrachteten Zeitraum hinweg zeigt sich allerdings eine deutliche Verringerung der Geschlechterunterschiede in den Arbeitswerten: Während zu Beginn der 1980er-Jahre noch signifikante Unterschiede bestehen, indem Männer mehr Wert auf gute Verdienstmöglichkeiten sowie hohe Aufstiegschancen legen und Frauen die Ausübung einer Tätigkeit mit helfendem Charakter höher schätzen, sind markante Geschlechterunterschiede im Jahr 2000 nur hinsichtlich der altruistischen Arbeitswerte zu beobachten. Trotz der in den 1980er- und 1990er-Jahren konstatierten Geschlechterunterschiede sind die Differenzen zwischen Männern und Frauen als eher gering einzuschätzen. Beide Geschlechter messen intrinsischen Aspekten wie der Ausübung einer interessanten und selbstbestimmten Tätigkeit eine höhere Bedeutung zu als den Verdienstmöglichkeiten und den Aufstiegschancen.

Anhand der vorliegenden Ergebnisse lässt sich die Leistungsfähigkeit der angebotsseitigen Erklärungsansätze von beruflicher Segregation einschätzen. Insgesamt deuten die Befunde der durchgeführten Analysen darauf hin, dass sich Geschlechtersegregation nur bedingt durch angebotsseitige Ansätze erklären lässt. Erstens sind die Gemeinsamkeiten zwischen Männern und Frauen bezüglich ihrer Arbeitswerte deutlich größer als die Unterschiede. Zweitens ist hinsichtlich der Arbeitswerte eine starke Angleichung zwischen den Geschlechtern im Zeitverlauf zu beobachten, wogegen das Ausmaß der beruflichen Segregation im gleichen Zeitraum so gut wie unverändert geblieben ist. Somit haben offenbar geschlechtsspezifische Veränderungen in den Arbeitswerten keine Auswirkungen auf Persistenz von beruflicher Segregation. Drittens zeigt sich ein nur schwacher Zusammenhang zwischen den Arbeitswerten der Erwerbstätigen und der Geschlechtstypik des ausgeübten Berufs, was darauf hindeutet, dass die Allokation in frauen- und männertypische Berufe nicht primär durch die Arbeitswerte geleitet wird.

\section{Executive summary}

Occupational sex segregation in the labour market is considered to be a fundamental cause of the gender wage gap. Due to occupational sex segregation, women more often hold jobs and occupations which yield low wages and offer only limited chances of promotion. There is ample research on the consequences of occupational sex segregation; however, its causes have attracted only little attention. Possible explanations of segregation and the resultant earnings differences between men and women can be found both on the demand-side and the supply-side of the labour market. On the demand-side, employers' preferences and discrimination may lead to segregation by allocating women to lower paying jobs as compared to men with identical qualifications. In contrast, supply-side explanations, such as those derived from neoclassical economic theory and sex-role socialisation, refer occupational sex segregation to gender differences in work values and preferences. According to the neoclassical economic theory, women anticipate unstable careers and choose jobs with family-friendly features which in turn yield lower wages. The sex-role socialisation model argues that differences in work values reflect traditional patterns of gender socialisation. Therefore, the analysis of gender differences in job values is crucial in evaluating demand-side explanations of occupational sex segregation. If there are significant gender differences in job preferences, then the disadvantages of women in the labour market in terms of income and occupational sex status is not a consequence of employer's discrimination, but results from their own occupational choice. Although the postulation of gender differences in job values is crucial to supply-side explanations of occupational sex segregation, this assumption has never been adequately tested for Germany.

Using ALLBUS data from 1980 to 2000, this study investigates the prevalence of, and changes in the job preferences of women and men. The respondents were asked to rate the importance of five job characteristics: high income, opportunity for advancement, interesting content, autonomy, opportunity to help others. Income and opportunity of advancement are usually classified as extrinsic rewards, whereas interesting content and autonomy are classified as intrinsic rewards, and opportunity to help represents altruistic rewards.

Our analysis shows that extrinsic rewards are more highly valued by men, while altruistic rewards are more valued by women. Over the time period covered in this study, the gen- 
der differences in work values have narrowed somewhat. In the early 1980s men attached more importance to income and chances of promotion than women did and women indicated a stronger preference to having opportunities to help others, whereas in 2000 we observe gender differences only with regard to altruistic job values. Despite a statistically significant gender gap in the 1980s and 1990s, the gender differences in job values have been rather small. Both men and women place greater value on intrinsic rewards, such as interesting job content and work autonomy than on income and chances of promotion.

The results presented here suggest that gender differences in preferences do not significantly contribute to explain occupational sex segregation in the labour market. First, we only find small gender differences and a high degree of similarity in the preferences of men and women. Second, the analysis shows that gender differences have narrowed over time, whereas the degree of occupational sex segregation has remained stable over the last two decades. Obviously, narrowing gender differences in job values had no effect on the persistence of segregation. Third, there is only a weak association between the work values held by men and women and the sex composition of their occupation, which indicates that the allocation of workers to male- and female-typical jobs is not primarily determined by the individuals' job preferences.

\section{Literatur}

Achatz, J.: Geschlechtersegregation im Arbeitsmarkt. In: Abraham, M., Hinz, T. (Hrsg.) Arbeitsmarktsoziologie. Probleme, Theorien, empirische Befunde, S. 263-301. VS Verlag, Wiesbaden (2005)

Achatz, J., Gartner, H., Glück, T.: Bonus oder Bias? Mechanismen geschlechtsspezifischer Entlohnung. Kölner Z. Soziol. Sozialpsychol. 57(3), 465-493 (2005)

Adler, M.A., Brayfield, A.: Women's work values in unified Germany. Regional differences as remnants of the past. Work Occup. 24(2), 245-266 (1997)

Aisenbrey, S., Brückner, H.: Occupational aspirations and the gender gap in wages. Eur. Sociol. Rev. 24(5), 633-649 (2008)

Allison, P.D., Christakis, N.A.: Logit models for sets of ranked items. Sociol. Methodol. 24, 199-228 (1994)

Allmendinger, J., Hinz, T.: Geschlechtersegregation in Organisationen und die Lohndifferenz zwischen Männern und Frauen. In: Gildemeister, R., Wetterer, A. (Hrsg.) Erosion oder Reproduktion geschlechtlicher Differenzierungen? Widersprüchliche Entwicklungen in professionalisierten Berufsfeldern und Organisationen, S. 172-188. Verlag Westfälisches Dampfboot, Münster (2007)

Becker, G.S.: Human capital. University of Chicago Press, New York (1964)

Becker, G.S.: A treatise on the family. Harvard University Press, Cambridge (1981)

Beutel, A.M., Marini, M.M.: Gender and values. Am Sociol. Rev. 60(3), 436-488 (1995)

Biersack, W., Parmentier, K., Schreyer, F.: Berufe im Spiegel der Statistik. Beschäftigung und Arbeitslosigkeit 1993-1999. Institut für Arbeitsmarkt- und Berufsforschung der Bundesanstalt für Arbeit, Nürnberg (2000)

Borg, I., Braun, M., Häder, M.: Arbeitswerte in Ost- und Westdeutschland: unterschiedliche Gewichte, aber gleiche Strukturen. ZUMANachr. 17(33), 65-82 (1993)

Braun, M., Borg, I.: Berufswerte im zeitlichen und im Ost-WestVergleich. In: Schmitt-Beck, R., Wasmer, M., Koch, A. (Hrsg.) Sozialer und politischer Wandel in Deutschland. Analysen mit ALLBUS-Daten aus zwei Jahrzehnten, S. 179-198. VS Verlag, Wiesbaden (2004)

Brückner, H.: Gender inequality in the life course. Social change and stability in West Germany, 1975-1995. De Gruyter, New York (2004)

Corcoran, M.E., Courant, P.N.: Sex-role socialisation and occupational segregation: an explanatory investigation. J. Post Keynesian Econ. 9(3), 330-346 (1987)

De Vaus, D., McAllister, I.: Gender and work orientation. Values and satisfaction in western Europe. Work Occup. 18(1), 72-93 (1991)

Dryler, H.: Parental role models, gender and educational choice. Br. J. Sociol. 49(3), 375-398 (1998)

England, P.: The failure of human capital theory to explain occupational sex segregation. J. Hum. Resour. 17(3), 358-370 (1982)

England, P.: Gender inequality in labor markets: the role of motherhood and segregation. Soc. Politics 12(2), 264-288 (2005)

Glass, J.: The impact of occupational segregation on working conditions. Soc. Forces 68(3), 779-796 (1990)

Gorman, E.H.: Marriage and money. The effect of marital status on attitudes towards pay and finances. Work Occup. 27(1), 64-88 (2000)

Habich, R.: Arbeitswerte, Arbeitsplatzrealität und Arbeitszufriedenheit. Ein Beitrag zum Problem der Anspruchsgewichtung bei der Erklärung von Arbeitszufriedenheit. Z. Soziol. 15(4), 278-294 (1986)

Hadjar, A.: Bildungsexpansion und Wandel von sozialen Werten. In: Hadjar, A., Becker, R. (Hrsg.) Die Bildungsexpansion. Erwartete und unerwartete Folgen, S. 205-230. VS Verlag, Wiesbaden (2006)

Halaby, C.N.: Where job values come from: family, schooling background, cognitive ability, and gender. Am. Sociol. Rev. 68(2), 251-278 (2003)

Hinz, T., Gartner, H.: Geschlechtsspezifische Lohnunterschiede in Branchen, Berufen und Betrieben. Z. Soziol. 34(1), 22-39 (2005)

Kalleberg, A.L., Loscocco, K.A.: Aging, values, and rewards: explaining age differences in job satisfaction. Am. Sociol. Rev. 48(1), 79-90 (1983)

Klein, M., Arzheimer, T.: Ranking- und Rating-Verfahren zur Messung von Wertorientierungen, untersucht am Beispiel des InglehartIndex. Empirische Befunde eines Methodenexperiments. Kölner Z. Soziol. Sozialpsychol. 51(3), 550-564 (1999)

Korupp, S.E., Sanders, K., Ganzeboom, H.B.G.: The intergenerational transmission of occupational status and sex-typing at children's labour market entry. Eur. J. Women's Stud. 9(1), 7-29 (2002)

Lee, K.S., Alwin, D.F., Tufis, P.A.: Beliefs about women's labour in the reunified Germany, 1991-2004. Eur. Sociol. Rev. 23(4), 487-503 (2007)

Liebeskind, U.: Arbeitsmarktsegregation und Einkommen. Vom Wert „weiblicher“ Arbeit. Kölner Z. Soziol. Sozialpsychol. 56(4), 630-652 (2004)

Long, J.S.: Regression models for categorical and limited dependent variables. Sage Publications, Thousand Oaks (1997)

Lorence, J.: Age differences in work involvement. Analyses of three explanations. Work Occup. 14(4), 533-557 (1987)

Mardsen, P.V., Kalleberg, A.L., Cook, C.R.: Gender differences in organizational commitment. Influences of work positions and gender roles. Work Occup. 20(3), 368-390 (1993)

Marini, M.M., Fan, P.L., Finley, E., Beutel, A.M.: Gender and job values. Sociol. Educ. 69(1), 49-65 (1996) 
Okamoto, D., England, P.: Is there a supply side to occupational sex segregation? Sociol. Perspect. 42(4), 557-582 (1999)

Padavic, I., Reskin, B.F.: Women and men at work. Pine Forge Press, Thousand Oaks (2002)

Parmentier, K., Schade, H.-J., Schreyer, F.: Berufsspezifische Strukturund Entwicklungsdaten 1980-1991: Erwerbsberufe im Spiegel der Statistik. Institut für Arbeitsmarkt- und Berufsforschung der Bundesanstalt für Arbeit, Nürnberg (1993)

Petersen, T., Saporta, I.: The opportunity structure for discrimination. Am. J. Sociol. 109(4), 852-901 (2004)

Rankin, W.L., Grube, J.W.: A comparison of ranking and rating procedures for value system measurement. Eur. J. Social Psychol. 10(3), 233-246 (1980)

Reskin, B.F.: Sex segregation in the workplace. Annu. Rev. Sociol. 19, 241-270 (1993)

Reskin, B.F., Bielby, D.D.: A sociological perspective on gender and career outcomes. J. Econ. Perspectives 19(1), 71-86 (2005)

Rosenfeld, R.A., Trappe, H.: Occupational sex segregation in state socialist and market economies: levels, patterns, and change in East and West Germany, 1980 and 1998. In: Leicht, K.T. (Hrsg.) The future of market transition. Elsevier, Amsterdam (2002)

Rowe, R., Snizek, W.E.: Gender differences in work values. Perpetuating the myth. Work Occup. 22(2), 215-229 (1995)

Russel, P.A., Gray, C.D.: Ranking or rating? Some data and their implications for the measurement of evaluative response. Br. J. Psychol. 85(1), 79-92 (1994)
Solga, H., Konietzka, D.: Das Berufsprinzip des deutschen Arbeitsmarktes: Ein geschlechtsneutraler Allokationsmechanismus? Schweiz. Z. Soziol. 26(1), 111-147 (2000)

Terwey, M.: ALLBUS: A German General Social Survey. Schmollers Jahrbuch. Z. Wirtsch. Sozialwiss. 120(1), 151-158 (2000)

Tolbert, P.S., Moen, P.: Men's and women's definitions of "good" jobs. Work Occup. 25(2), 168-194 (1998)

Trappe, H.: Berufliche Segregation im Kontext. Über die Folgen geschlechtsspezifischer Berufsentscheidungen in Ost- und Westdeutschland. Kölner Z. Soziol. Sozialpsychol. 58(1), 50-78 (2006)

Waite, L.J., Berryman, S.E.: Women in nontraditional occupations: choice and turnover. Rand Corporation, Santa Monica (1985)

Wright, J.D., Hamilton, R.F.: Work satisfaction and age: some evidence for the "job change" hypothesis. Soc. Forces 56(4), 1140-1158 (1978)

Matthias Pollmann-Schult, Studium der Soziologie an der Freien Universität Berlin, 2000 Abschluss als Diplom-Soziologe. Von 20002003 wissenschaftlicher Mitarbeiter am Max-Planck-Institut für Bildungsforschung, Berlin, 2003 Promotion zum Dr. phil. Seit 2004 wissenschaftlicher Assistent an Fakultät für Soziologie der Universität Bielefeld.

Forschungsfelder: Fehlallokation im Arbeitsmarkt, Erwerbsverhalten im Haushaltskontext, Erwerbswünsche von Vätern.

E-Mail: matthias.pollmann-schult@uni-bielefeld.de 